\title{
Non-uniqueness of minimizers for strictly polyconvex functionals
}

\author{
Spadaro, E N
}

Abstract: In this note we solve a problem posed by Ball (in Philos Trans R Soc Lond Ser A 306(1496):557611,1982 ) about the uniqueness of smooth equilibrium solutions to boundary value problems for strictly polyconvex functionals,

$$
\mathcal{F}(u)=\int_{\Omega} f(\nabla u(x)) \mathrm{d} x \quad \text { and }\left.\quad u\right|_{\partial \Omega}=u_{0},
$$

where $\Omega$ is homeomorphic to a ball. We give several examples of non-uniqueness. The main example is a boundary value problem with at least two different global minimizers, both analytic up to the boundary. All these examples are suggested by the theory of minimal surfaces.

DOI: https://doi.org/10.1007/s00205-008-0156-y

Posted at the Zurich Open Repository and Archive, University of Zurich ZORA URL: https://doi.org/10.5167/uzh-18889

Journal Article

Published Version

Originally published at:

Spadaro, E N (2009). Non-uniqueness of minimizers for strictly polyconvex functionals. Archiv for Rational Mechanics and Analysis, 193(3):659-678.

DOI: https://doi.org/10.1007/s00205-008-0156-y 


\title{
Non-Uniqueness of Minimizers for Strictly Polyconvex Functionals
}

\author{
Emanuele Nunzio Spadaro
}

Communicated by S. MÜLLER

\begin{abstract}
In this note we solve a problem posed by Ball (in Philos Trans R Soc Lond Ser A 306(1496):557-611, 1982) about the uniqueness of smooth equilibrium solutions to boundary value problems for strictly polyconvex functionals,

$$
\mathcal{F}(u)=\int_{\Omega} f(\nabla u(x)) \mathrm{d} x \text { and }\left.u\right|_{\partial \Omega}=u_{0},
$$

where $\Omega$ is homeomorphic to a ball.

We give several examples of non-uniqueness. The main example is a boundary value problem with at least two different global minimizers, both analytic up to the boundary. All these examples are suggested by the theory of minimal surfaces.
\end{abstract}

\section{Introduction}

In [3] Ball posed the following problem (Problem 8). Let $\mathcal{F}$ be a strictly polyconvex functional,

$$
\mathcal{F}(u)=\int_{\Omega} f(\nabla u(x)) \mathrm{d} x,
$$

where $u: \Omega \subset \mathbb{R}^{n} \rightarrow \mathbb{R}^{m}(n, m \geqq 2)$ is in some Sobolev space and $\Omega$ is homeomorphic to a ball. Given pure displacement boundary conditions $\left.u\right|_{\partial \Omega}=u_{0}$, is there uniqueness of smooth equilibrium solutions?

We answer this question negatively, giving an example in dimension $2(m=$ $n=2$ ) of a smooth integrand $f$ and a boundary value $u_{0}$ such that (1.1) admits at least two absolute minimizers, both analytic up to the boundary. We state this result in the following theorem. 
Theorem 1.1. Let $\Omega \subset \mathbb{R}^{2}$ be the unit ball. There is a strictly polyconvex function $f: \mathbb{R}^{4} \rightarrow \mathbb{R}$ and an analytic function $u_{0}$ on $\partial \Omega$ such that the functional $\mathcal{F}$ has at least two analytic (up to the boundary) absolute minimizers in

$$
H=\left\{u \in W^{1,2}\left(\Omega ; \mathbb{R}^{2}\right):\left.u\right|_{\partial \Omega}=u_{0}\right\} .
$$

This example is taken from the elegant proof of non-uniqueness of the Dirichlet problem for the non-parametric area functional in codimension 2, given by Lawson and Osserman in [13].

Roughly speaking, we choose boundary conditions corresponding to squeezing the ball until it has a dumb-bell shape. Then we prove that an absolute minimizer cannot have the symmetries of the dumb-bell, thus implying non-uniqueness. In dimension two, the boundary data we construct is not one to one with its image. The reason is that, in order to create the constraint for which the minimizer is not symmetric, we have to overlap the two halves of the dumb-bell. Nevertheless, we show that the lack of injectivity is not the main point of the construction. In the same spirit as in the previous example, we describe an injective boundary displacement in dimension 3 , that is a function $u_{0}: \partial \Omega \subset \mathbb{R}^{2} \rightarrow \mathbb{R}^{3}$, for which the same conclusion as in Theorem 1 holds.

Theorem 1.1'. There is a strictly polyconvex function $f: \mathbb{R}^{6} \rightarrow \mathbb{R}$ and an analytic injective function $u_{0}: \partial \Omega \rightarrow \mathbb{R}^{3}$, where $\Omega \subset \mathbb{R}^{2}$ is the unit ball, such that the functional $\mathcal{F}$ has at least two absolute minimizers, analytic up to the boundary, with boundary value $u_{0}$.

The injectivity of the boundary value is significant in reading this example in Elasticity Theory: indeed, one can imagine that the displacement given in the theorem corresponds to a physically admissible deformation of a planar membrane in $\mathbb{R}^{3}$, without overlapping, and that the functional is the elastic energy of the body (even if frame indifference and polyconvexity are not very compatible for maps from $\mathbb{R}^{2}$ to $\mathbb{R}^{3}$, as shown by Pipkin's work on membranes [16]).

However, it is worthwhile to stress two limits of our counterexample in Theorem 1.1. It is genuinely two dimensional and (see also Remark 2.2) the Jacobian of minimizing planar maps changes sign: unlike the lack of injectivity, we believe this restriction plays a role in the construction of the counterexample.

Starting from this, we construct examples with arbitrarily many local minimizers and an example with uncountably many stable stationary points (equilibrium solutions), as stated in the following theorems.

Theorem 1.2. For every $n \in \mathbb{N}$, there exist a smooth planar domain $\Omega$ homeomorphic to a ball, a smooth function $\phi: \partial \Omega \rightarrow \mathbb{R}^{2}$ and a smooth strictly polyconvex functional $\mathcal{F}$ with at least $n$ local minimizers in the class $u \in W^{1,2}\left(\Omega ; \mathbb{R}^{2}\right)$ with $\left.u\right|_{\partial \Omega}=\phi$.

Theorem 1.3. There exist a planar domain $\Omega$ homeomorphic to a ball (with $\partial \Omega$ lipschitz except at one point), a function $\phi: \partial \Omega \rightarrow \mathbb{R}^{2}$ and a strictly polyconvex function $f$ such that the functional $\mathcal{F}$ has uncountably many stable equilibrium solutions in the class $u \in W^{1,2}\left(\Omega ; \mathbb{R}^{2}\right)$ with $\left.u\right|_{\partial \Omega}=\phi$. 
The idea for these constructions comes again from the theory of minimal surfaces: we indeed apply the so called "bridge principle" to connect local minimizers and to build new ones (for a proof of a general bridge principle for minimal surfaces, and for an account of its history, we refer to the paper of B. White [22]).

As for Theorem 1, we point out that one can have injectivity of the boundary displacement in both Theorems 1.2 and 1.3 if the target is $\mathbb{R}^{3}$, see Remarks 3.5 and 3.6.

The problem of uniqueness for equilibrium solutions in nonlinear elasticity has received much well-deserved attention in the last decades. There are, indeed, results both in the direction of uniqueness and in that of non-uniqueness. Among the first, we recall the uniqueness result of John [10] in the case of deformations with small strains; the result of Knops and Stuart [11] in which they prove uniqueness for linear boundary displacements and $\Omega$ star-shaped; and the work of Taheri [20]. On the other side, non-uniqueness has been proved for mixed boundary value problems, see for example [2]; for domains not homeomorphic to a ball, see for example the works of John [10], Post and Sivaloganathan [17] and Taheri [21], in which nonuniqueness is proved when the domain $\Omega$ is topologically non-trivial (meaning, for $\Omega \subset \mathbb{R}^{2}$, that $\Omega$ is not simply connected); and, finally, for non-smooth equilibrium solutions, see for example the works of Müller and Šverák [15] and of Székelyhidi [18].

This paper is organized as follows. In Section 2 we report a more elementary version of the proof of Lawson and Osserman in [13] and prove Theorems 1.1 and 1.1'. In Section 3 we prove Theorems 1.2 and 1.3: to this aim, we state and prove an elementary "bridge principle" which is sufficient for our purposes.

We would like to thank C. De Lellis, B. Kirchheim, J. Kristensen and L. Székelyhidi for many useful discussions; and N. Fusco for having posed the question about the injectivity of the boundary value.

\section{Existence of at least two analytic different minimizers}

Throughout this section, we denote by $\Omega$ the unit ball in $\mathbb{R}^{2}$,

$$
\Omega=\left\{x=\left(x_{1}, x_{2}\right) \in \mathbb{R}^{2} \mid x_{1}^{2}+x_{2}^{2}<1\right\},
$$

and by $\mathcal{A}$ the area functional,

$$
\mathcal{A}(u)=\int_{\Omega} \sqrt{1+|\nabla u(x)|^{2}+(\operatorname{det} \nabla u(x))^{2}} \mathrm{~d} x .
$$

Here $u$ belongs to the Sobolev space $W^{1,2}\left(\Omega ; \mathbb{R}^{2}\right)$ and $\mathcal{A}$ is the area of the graph of $u$.

In [13] Lawson and Osserman proved the existence part of the following result.

Theorem 2.1. There exists a real analytic function $u_{0}: \partial \Omega \rightarrow \mathbb{R}^{2}$ such that $\mathcal{A}$ has at least two absolute analytic (up to the boundary) minimizers in the class

$$
H=\left\{u \in W^{1,2}\left(\Omega ; \mathbb{R}^{2}\right):\left.u\right|_{\partial \Omega}=u_{0}\right\} .
$$




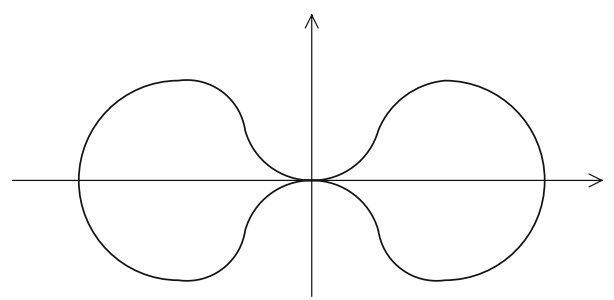

Fig. 1. Shape of the curve $\gamma_{0}$

Here we report a modified and more elementary version of their proof.

Proof. We divide the proof into steps.

STEP 1 . We define the boundary condition $u_{0}$.

Let $\gamma_{0}$ be an analytic dumb-bell shaped (immersed) curve in $\mathbb{R}^{2}$ of the type pictured in Fig. 1: $\gamma_{0}$ is assumed to be invariant under the symmetries with respect to the two axes,

$$
\left(x_{1}, x_{2}\right) \mapsto\left(-x_{1}, x_{2}\right) \text { and }\left(x_{1}, x_{2}\right) \mapsto\left(x_{1},-x_{2}\right)
$$

(It is not difficult to see that such curves exist: an easy example is the curve defined by the equation $y^{2}-x^{4}+x^{6}=0$ ).

Define $u_{0}: \partial \Omega \rightarrow \mathbb{R}^{2}$ to be a parametrized double tracing of $\gamma=R \gamma_{0}$ (we defer the choice of $R$ until later), where each loop is covered once in each direction and the parameter is a multiple of the arc length:

for example, set $u_{0}$ to be the parametrization such that

$$
u_{0}(1,0)=u_{0}(0,1)=u_{0}(-1,0)=u_{0}(0,-1)=0,
$$

and maps each half of $\partial \Omega$ onto $\gamma$, as sketched in the picture (Fig. 2).

Step 2. Assuming uniqueness of the minimizer, we estimate its energy from below, proving that

$$
\mathcal{A}\left(u_{\min }\right) \geqq 4 c_{0} R^{2},
$$

where $c_{0}$ is a constant depending on $\gamma_{0}$ and $R$ is the parameter defining $\gamma$, hence the boundary condition $u_{0}$.

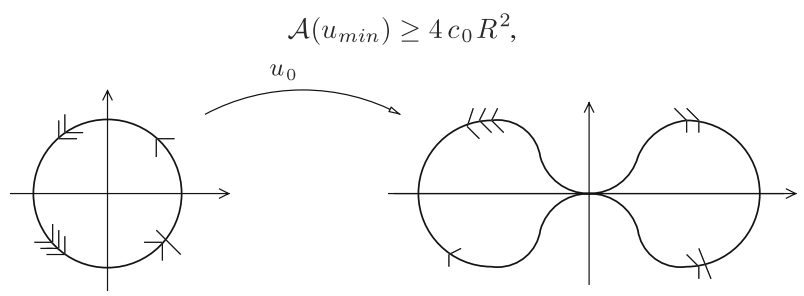

Fig. 2. The boundary value $u_{0}$ 
Due to the analyticity of $\gamma$ and to its symmetries, it follows that the graph of $u_{0}$, that is the set

$$
\Gamma_{u_{0}}=\left\{\left(x, u_{0}(x)\right) \mid x \in \partial \Omega\right\},
$$

is an analytic Jordan curve in $\mathbb{R}^{4}$ and is invariant under the following symmetries $\sigma_{1}, \sigma_{2}: \mathbb{R}^{4} \rightarrow \mathbb{R}^{4}$

$$
\begin{aligned}
& \sigma_{1}\left(x_{1}, x_{2}, x_{3}, x_{4}\right)=\left(-x_{1},-x_{2}, x_{3},-x_{4}\right), \\
& \sigma_{2}\left(x_{1}, x_{2}, x_{3}, x_{4}\right)=\left(-x_{1}, x_{2},-x_{3}, x_{4}\right) .
\end{aligned}
$$

From the theory of generalized parametric minimal surfaces we know that there exists a minimizer of $\mathcal{A}$ in $H$ (the so called Douglas solution), see [13] (Theorem 4.1, pag. 5), and that this minimizer is analytic up to the boundary, see [9], (for a comprehensive account of these results we refer to [4] - in particular, for the regularity see Theorem 2 of Section 7.3, p. 33, vol II).

Let us assume the uniqueness of such a minimizer $u_{\min }$ : it follows that its graph must be invariant under the symmetries $\sigma_{1}$ and $\sigma_{2}$, that is

$$
\begin{aligned}
u_{\min }\left(-x_{1},-x_{2}\right) & =\left(u_{\min }^{1}\left(-x_{1},-x_{2}\right), u_{\min }^{2}\left(-x_{1},-x_{2}\right)\right) \\
& =\left(u_{\min }^{1}\left(x_{1}, x_{2}\right),-u_{\min }^{2}\left(x_{1}, x_{2}\right)\right), \\
u_{\min }\left(-x_{1}, x_{2}\right) & =\left(u_{\min }^{1}\left(-x_{1}, x_{2}\right), u_{\min }^{2}\left(-x_{1}, x_{2}\right)\right) \\
& =\left(-u_{\min }^{1}\left(x_{1}, x_{2}\right), u_{\min }^{2}\left(x_{1}, x_{2}\right)\right) .
\end{aligned}
$$

From this we easily deduce the following two consequences:

(i)

$$
u_{\min }(t, 0)=0 \text { and }\left\{\begin{array}{l}
u_{\min }^{1}(0, t)=0, \\
u_{\min }^{2}(0, t)=u_{\min }^{2}(0,-t),
\end{array}\right.
$$

for every $t \in(-1,1)$, by direct substitution in (2.2);

(ii) if we set $\Omega_{i}=\Omega \cap\{i$ th - quadrant $\}$ for $i=1, \ldots, 4$, that is

$$
\begin{aligned}
& \Omega_{1}=\Omega \cap\left\{\left(x_{1}, x_{2}\right) \mid x_{1}>0, x_{2}>0\right\}, \\
& \Omega_{2}=\Omega \cap\left\{\left(x_{1}, x_{2}\right) \mid x_{1}<0, x_{2}>0\right\}, \quad \text { etc } \ldots,
\end{aligned}
$$

we have

$$
\begin{aligned}
\mathcal{A}\left(u_{\min }, \Omega_{i}\right) & :=\int_{\Omega_{i}} \sqrt{1+\left|\nabla u_{\min }(x)\right|^{2}+\left(\operatorname{det} \nabla u_{\min }(x)\right)^{2}} \mathrm{~d} x \\
& =\mathcal{A}\left(u_{\min }, \Omega_{j}\right), \quad \forall i, j \in\{1, \ldots, 4\} .
\end{aligned}
$$


If we call $A$ the subset of the plane enclosed by one bubble of $\gamma$ (say, for example, the shaded region in the right portion of Fig. 3), we claim that

$$
\mathcal{A}\left(u_{\min }, \Omega_{i}\right) \geqq \mathcal{L}^{2}(A)=c_{0} R^{2}, \quad \forall i=1, \ldots, 4,
$$

where $c_{0}>0$ is a constant depending on $\gamma_{0}$ and $\mathcal{L}^{2}$ is the Lebesgue measure in the plane, and hence (2.1).

Without loss of generality (see (ii) above), we consider $i=1$ and calculate the degree of the map $u_{\min }$ in $\Omega_{1}$ at a point $y \in A$ (for a definition and the first properties of the degree of a continuous map we refer to [14]). We claim that it different from 0: indeed, from (2.3) we infer that $\left.u_{\min }\right|_{\partial \Omega_{1}}$ is homotopic to the map

$$
v= \begin{cases}u_{\min } & \text { in } \Omega_{1} \cap\left\{x_{1}^{2}+x_{2}^{2}=1\right\} \\ 0 & \text { in } \Omega_{1} \cap\left(\left\{x_{1}=0\right\} \cup\left\{x_{2}=0\right\}\right),\end{cases}
$$

for which $\operatorname{deg}\left(v, \Omega_{1}, y\right)=1$, as can be seen by an easy computation. So, one infers immediately that $A \subseteq u_{\min }\left(\bar{\Omega}_{1}\right)$, hence

$$
\begin{aligned}
\mathcal{A}\left(u_{\min }, \Omega_{1}\right) & =\int_{\Omega_{1}} \sqrt{1+\left|\nabla u_{\min }(x)\right|^{2}+\left(\operatorname{det} \nabla u_{\min }(x)\right)^{2}} \mathrm{~d} x \\
& \geqq \int_{\Omega_{1}}\left|\operatorname{det} \nabla u_{\min }(x)\right| \mathrm{d} x \geqq \mathcal{L}^{2}\left(u_{\min }\left(\bar{\Omega}_{1}\right)\right) \geqq \mathcal{L}^{2}(A)=c_{0} R^{2} .
\end{aligned}
$$

This concludes the proof of (2.4).

SteP 3. If $R$ is sufficiently large, we exhibit another function $u \in H$ such that $\mathcal{A}(u)<\mathcal{A}\left(u_{\min }\right)$, thus contrasting with the assumption of $u_{\min }$ being the unique minimizer.

Consider the function $u$ which is linear on the segments

$$
\left\{\left(x_{1}, x_{2}\right) \in \Omega \mid x_{1}+x_{2}=c\right\},
$$

and such that $\left.u\right|_{\partial \Omega}=u_{0}$. Note that $u$ is constant on these segments if $|c| \leqq 1$ (Fig. 3). It is easy to recognize that

$$
\begin{aligned}
\mathcal{A}(u) & =\int_{\Omega} \sqrt{1+|\nabla u(x)|^{2}+(\operatorname{det} \nabla u(x))^{2}} \mathrm{~d} x \\
& \leqq \int_{\Omega}(1+|\nabla u(x)|+|\operatorname{det} \nabla u(x)|) \mathrm{d} x \\
& \leqq \pi+c_{1} R+2 \mathcal{L}^{2}(A)=\pi+c_{1} R+2 c_{0} R^{2},
\end{aligned}
$$

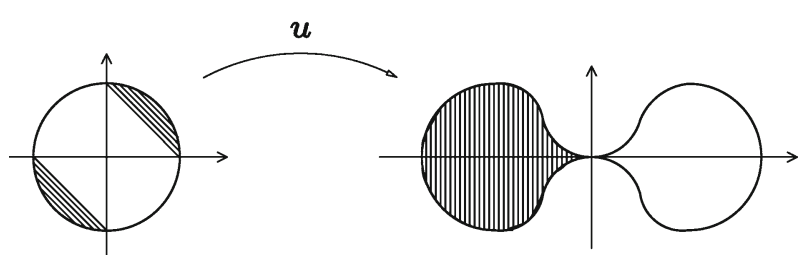

Fig. 3. The competitor $u$ 
with $c_{1}$ a constant depending on $\gamma_{0}$, where we used the injectivity of $u$ in the set $\left\{\left(x_{1}, x_{2}\right) \in \Omega|| x_{1}+x_{2} \mid>1\right\}$ and the area formula.

For $R$ large enough, we have

$$
2 c_{0} R^{2}>\pi+c_{1} R .
$$

Therefore, we conclude that $\mathcal{A}(u)<\mathcal{A}\left(u_{\text {min }}\right)$, that is the minimizer for $\mathcal{A}$ in $\mathrm{H}$ is not unique. Hence, there are at least two absolute minimizers which, for the regularity theorem quoted before, [9], are analytic up to the boundary.

The preceding theorem provides a smooth polyconvex functional with at least two analytic minimizers: however, the area functional is not strictly polyconvex, according to the following definition.

Definition 2.1. A function $f: \mathbb{R}^{4} \rightarrow \mathbb{R}$ is strictly polyconvex if there exists a positive constant $c>0$ with the following property:

for every $x \in \mathbb{R}^{4}$ there exist $v \in \mathbb{R}^{4}$ and $a \in \mathbb{R}$ such that

$$
f(y) \geqq f(x)+v \cdot(y-x)+a(\operatorname{det} y-\operatorname{det} x)+c|y-x|^{2} \quad \forall y \in \mathbb{R}^{4},
$$

where det $x=x_{1} x_{4}-x_{2} x_{3}$.

Or, equivalently, $f$ is polyconvex if there exists a convex function $g: \mathbb{R}^{5} \rightarrow \mathbb{R}$ and $c>0$ such that

$$
f(x)=c|x|^{2}+g(x, \operatorname{det} x) .
$$

Remark 2.1. It is not difficult to show the equivalence of the two definitions: the second condition implies the first because of the convexity of $g$; the converse follows by the observation that $f(x)-c|x|^{2}$ is by hypothesis a convex function in $(x, \operatorname{det} x)$.

Due to the regularity of the minimizers (they are analytic, hence in particular they are globally Lipschitz), it is a simple task to modify the area functional in order to get a strictly polyconvex one with two global minimizers.

Proof of Theorem 1.1. Let $u_{\mathrm{min}}$ and $v_{\min }$ be the two analytic global minimizers for $\mathcal{A}$ given by Theorem 2.1 and consider a smooth positive convex function $g: \mathbb{R} \rightarrow \mathbb{R}$ such that

$$
\begin{array}{r}
g(x)=0 \text { if }|x| \leqq 2 \lambda, \\
x^{2} \leqq g(x) \leqq 2 x^{2} \text { if }|x| \geqq 4 \lambda,
\end{array}
$$

where $\lambda=\max \left\{\left\|\nabla u_{\min }\right\|_{L^{\infty}(\Omega)},\left\|\nabla v_{\min }\right\|_{L^{\infty}(\Omega)}\right\}$. Then the functional

$$
\mathcal{G}(u)=\mathcal{A}(u)+\int_{\Omega} g(|\nabla u(x)|) \mathrm{d} x
$$

is a smooth strictly polyconvex functional (as can be verified easily) and has $u_{\text {min }}$ and $v_{\min }$ as minimizers: indeed,

$$
\mathcal{G}\left(u_{\min }\right)=\mathcal{A}\left(u_{\min }\right) \leqq \mathcal{A}(u) \leqq \mathcal{G}(u),
$$

for every $u \in H$ (the same being true for $v_{\min }$ ).

Hence, we have proved Theorem 1.1. 
Remark 2.2. We note here that, for every $u: \Omega \rightarrow \mathbb{R}^{2}$ sufficiently smooth and such that $\left.u\right|_{\partial \Omega}=u_{0}$ (hence, in particular, for the analytic minimizers), one can prove that

$$
\int_{\Omega} \operatorname{det}(\nabla u(x)) \mathrm{d} x=0
$$

that is $u$ is not sign-preserving.

To see this, write $\operatorname{det}(\nabla u)$ as the divergence of a vector,

$$
\operatorname{det}(\nabla u)=\frac{\partial u^{1}}{\partial x_{1}} \frac{\partial u^{2}}{\partial x_{2}}-\frac{\partial u^{1}}{\partial x_{2}} \frac{\partial u^{2}}{\partial x_{1}}=\operatorname{div}\left(u^{2}\left(\nabla u^{1}\right)^{\perp}\right)
$$

where $\left(\nabla u^{1}\right)^{\perp}=\left(-\frac{\partial u^{1}}{\partial x_{2}}, \frac{\partial u^{1}}{\partial x_{1}}\right)$; and use the divergence theorem to compute

$$
\begin{aligned}
\int_{\Omega} \operatorname{det}(\nabla u(x)) \mathrm{d} x & =\int_{\Omega} \operatorname{div}\left(u^{2}(x)\left(\nabla u^{1}(x)\right)^{\perp}\right) \mathrm{d} x \\
& =\int_{\partial \Omega} u^{2}(x)\left(\nabla u^{1}(x)\right)^{\perp} \cdot \eta \mathrm{d} x \\
& =-\int_{\partial \Omega} u^{2}(x) \nabla u^{1}(x) \cdot \tau \mathrm{d} x \\
& =-\int_{0}^{2 \pi} u_{0}^{2}(s)\left(u_{0}^{1}\right)^{\prime}(s) \mathrm{d} s,
\end{aligned}
$$

where we considered $u_{0}$ as a function defined in $[0,2 \pi], u_{0}:[0,2 \pi] \rightarrow \mathbb{R}^{2}$ and $\eta$ and $\tau$ are, respectively, the normal and the tangential vectors to $\partial \Omega$, that is

$$
\eta\left(x_{1}, x_{2}\right)=\left(x_{1}, x_{2}\right) \quad \text { and } \quad \tau\left(x_{1}, x_{2}\right)=\left(x_{2},-x_{1}\right) .
$$

Now, we notice that the symmetries of $\gamma$ and the choice of the parametrization $u_{0}$ imply that, for $s \in\left[0, \frac{\pi}{2}\right]$,

$$
\left\{\begin{array} { l } 
{ u _ { 0 } ^ { 1 } ( s ) = - u _ { 0 } ^ { 1 } ( \frac { 3 \pi } { 2 } + s ) } \\
{ u _ { 0 } ^ { 2 } ( s ) = u _ { 0 } ^ { 2 } ( \frac { 3 \pi } { 2 } + s ) }
\end{array} \text { and } \left\{\begin{array}{l}
u_{0}^{1}\left(\frac{\pi}{2}+s\right)=-u_{0}^{1}(\pi+s) \\
u_{0}^{2}\left(\frac{\pi}{2}+s\right)=u_{0}^{2}(\pi+s) .
\end{array}\right.\right.
$$

Hence, we have

$$
\int_{0}^{\frac{\pi}{2}} u_{0}^{2}\left(u_{0}^{1}\right)^{\prime}=-\int_{\frac{3 \pi}{2}}^{2 \pi} u_{0}^{2}\left(u_{0}^{1}\right)^{\prime} \text { and } \int_{\frac{\pi}{2}}^{\pi} u_{0}^{2}\left(u_{0}^{1}\right)^{\prime}=-\int_{\pi}^{\frac{3 \pi}{2}} u_{0}^{2}\left(u_{0}^{1}\right)^{\prime},
$$

so that, from (2.8), we conclude (2.7). Moreover, it is not difficult to see that, for all such regular maps $u$ with $\left.u\right|_{\partial \Omega}=u_{0}$,

$$
\operatorname{det}(\nabla u) \not \equiv 0 .
$$

To see (2.9), we prove that $u(\Omega)$ has positive measure, which, together with Sard's lemma, implies (2.9), that is, that not all the points in $\Omega$ can be critical. More precisely, we claim that $u(\Omega)$ contains one of the two bounded connected components delimited by $\gamma$, which we call $A$ and $B$ (see Fig. 4 ). 


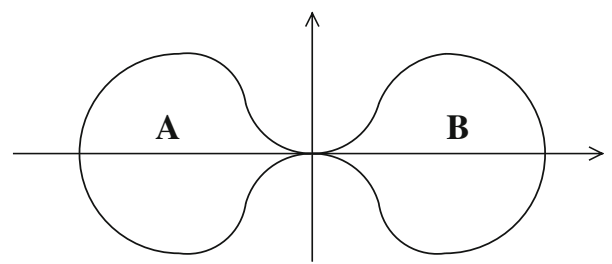

Fig. 4. $A$ and $B$

Indeed, the claim follows once one proves that, for every pair of points $x \in A$ and $y \in B, \gamma$ is not homotopic to a constant in $\mathbb{R}^{2} \backslash\{x, y\}$. In turn, the last assertion is a consequence of the following three well known facts:

(i) there exists a continuous retraction of $\mathbb{R}^{2} \backslash\{x, y\}$ into the image of $\gamma, \gamma(\partial \Omega)$, which is a topological rose (or bouquet of circles);

(ii) the loop $\gamma$ is homotopic to $a b a^{-1} b^{-1}$, where $a$ and $b$ are the generators of the first homotopy group of this space corresponding to parametrize clockwise $\partial A$ and $\partial B$

(iii) the first homotopic group of a topological rose with more than one circle is not commutative.

Let us point out the role of the boundary condition $u_{0}$ in the construction above: thanks to its symmetries we can rule out the uniqueness of the minimizer.

The lack of injectivity of the boundary displacement is used only to provide the right symmetries: indeed, if we consider functions $u: \Omega \rightarrow \mathbb{R}^{3}$, it is possible to find an example with one to one boundary conditions.

The example is based again on the area functional, and the proof is similar to the one of Theorem 2.1 (and can be found in [13] as well): we sketch it here.

Proof of Theorem 1.1'. We consider the curve $\gamma_{0}$ of the type pictured in Fig. 5. It is assumed to:

$(\alpha)$ be analytic,

( $\beta$ ) lie in the union of two pairs of parallel planes, each pair at distance $\varepsilon \ll 1$ apart,

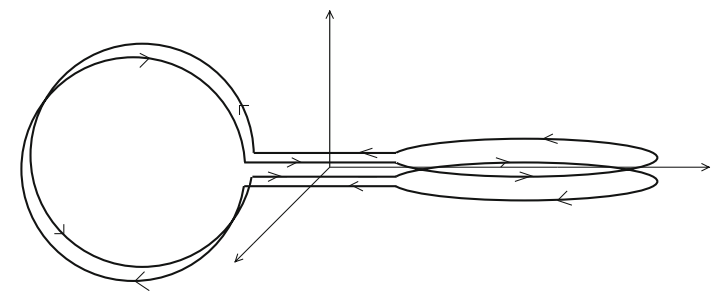

Fig. 5. Shape of the curve $\gamma_{0}$ in the three dimensional case 
$(\gamma)$ be invariant with respect to the following symmetries (note that the first two do not preserve the orientation of $\gamma_{0}$ ):

$$
\begin{aligned}
& \left(x_{1}, x_{2}, x_{3}\right) \mapsto\left(x_{1}, x_{2},-x_{3}\right), \\
& \left(x_{1}, x_{2}, x_{3}\right) \mapsto\left(x_{1},-x_{2}, x_{3}\right), \\
& \left(x_{1}, x_{2}, x_{3}\right) \mapsto\left(-x_{1},-x_{3}, x_{2}\right) .
\end{aligned}
$$

As a boundary condition we consider $u_{0}: \partial \Omega \rightarrow \mathbb{R}^{3}$ a parametrization of $\gamma=R \gamma_{0}$, where the parameter is a multiple of the arc length, so that the graph of $u_{0}$ is symmetric with respect to the following symmetries:

$$
\begin{aligned}
& \sigma_{1}\left(x_{1}, x_{2}, x_{3}, x_{4}, x_{5}\right)=\left(-x_{1},-x_{2}, x_{3},-x_{4},-x_{5}\right), \\
& \sigma_{2}\left(x_{1}, x_{2}, x_{3}, x_{4}, x_{5}\right)=\left(-x_{1}, x_{2},-x_{3},-x_{4},-x_{5}\right), \\
& \sigma_{3}\left(x_{1}, x_{2}, x_{3}, x_{4}, x_{5}\right)=\left(x_{2},-x_{1},-x_{3}, x_{5},-x_{4}\right) .
\end{aligned}
$$

If the minimizer were unique, its energy (which is the area of its graph) would be at least $4 c_{0} R^{2}$, where $c_{0}$ is a constant depending only on $\gamma_{0}$ (here one can use a degree argument as in the proof of Theorem 2.1, or one can see the original proof in [13] - this symmetric minimizer must resemble the function pictured in Fig. 6 which is invariant under the symmetries above). But the competitor sketched in Fig. 7 is easily recognized to have energy less than $\left[2 c_{0}+c_{1} \varepsilon\right] R^{2}+c_{2} R+c_{3}$, so that we reach the same contradiction for $R$ large enough and $\varepsilon \ll 1$. This concludes the proof of Theorem 1.1'.

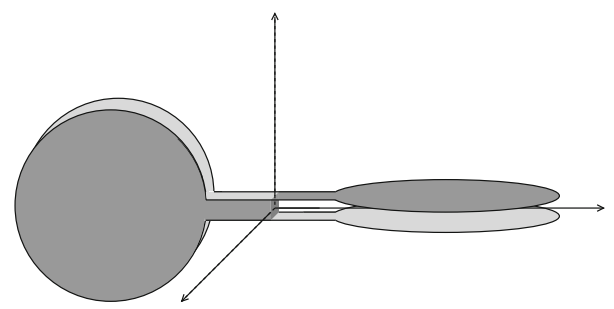

Fig. 6. Symmetric function

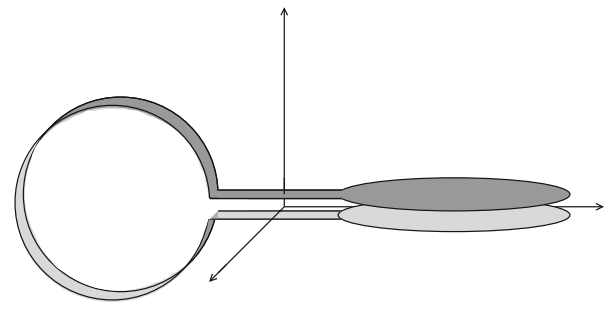

Fig. 7. Competitor 


\section{Arbitrarily many local minimizers and uncountable stable equilibrium solutions}

Starting from the example of Theorem 1.1, we construct boundary value problems with more than two equilibrium solutions.

As pointed out in the introduction, the basic idea in these constructions is to use a "bridge principle", as for minimal surfaces: the main step for this procedure is given in the next theorem. In what follows $f$ will always denote a smooth strictly polyconvex function, $f: \mathbb{R}^{2 d} \rightarrow \mathbb{R}$, such that there exist constants $c$ and $C$ for which

$$
c|x|^{2} \leqq f(x) \leqq C|x|^{2}
$$

and, for every planar domain $\Omega$, we set $\mathcal{F}(\cdot, \Omega)$ to be the functional

$$
\mathcal{F}(u, \Omega):=\int_{\Omega} f(\nabla u(x)) \mathrm{d} x .
$$

For the sake of clarity, $f: \mathbb{R}^{2 d} \rightarrow \mathbb{R}$ is strictly polyconvex if there exists a convex function $g: \mathbb{R}^{\frac{d(d+3)}{2}} \rightarrow \mathbb{R}$ such that

$$
f(x)=g\left(x, M_{1}(x), \ldots, M_{\frac{d(d-1)}{2}}(x)\right)+c|x|^{2},
$$

where $M_{i}(x)$ are the Minors of the $d \times 2$ matrix $x$. In the case $d=2$ there is only one such Minor, the determinant of $x$, and we recover the definition given in the previous section. For $d=3$ there are three Minors,

$$
M_{1}(x)=x_{1} x_{4}-x_{2} x_{3}, \quad M_{2}(x)=x_{1} x_{6}-x_{2} x_{5}, \quad M_{3}(x)=x_{2} x_{6}-x_{3} x_{5} .
$$

Remark 3.1. The first inequality of (3.1) is not restrictive. Consider indeed a strictly polyconvex function $f$. Then

$$
f(x) \geqq f(0)+C_{0} \cdot x+\sum_{i=1}^{\frac{d(d-1)}{2}} C_{i} M_{i}(x)+c|x|^{2} .
$$

If we consider

$$
g(x):=f(x)-f(0)-C_{0} \cdot x-\sum_{i=1}^{\frac{d(d-1)}{2}} C_{i} M_{i}(x),
$$

the minimizers of $\int_{\Omega} f(\nabla u)$ and those of $\int_{\Omega} g(\nabla u)$ coincide, because $f(0)+$ $C_{0} \cdot x+\sum_{i=1}^{\frac{d(d-1)}{2}} C_{i} M_{i}(x)$ is a Null-Lagrangian, and $g$ satisfies the left hand side inequality of (3.1). 
Before stating and proving the theorem, we introduce some additional notation (consistent with that used in [22]).

Given two planar smooth open sets with disjoint closures, $O_{1}$ and $O_{2}$, we say that a smooth curve $\Gamma$ connects them transversally if $\Gamma \cap O_{i} \subset \partial \Gamma$ for $i=1,2$ and $\Gamma$ is not tangent to $\partial O_{1}$ and $\partial O_{2}$.

Moreover, given two such open sets and a curve connecting them transversally, we say that $P \subset \mathbb{R}^{2}$ is a bridge along $\Gamma$ if it is the image of an homeomorphism

$$
\psi:(-1,1) \times[-1,1] \rightarrow P
$$

such that $\psi(0, \cdot)$ parametrizes $\Gamma$ and such that $\psi(x, t) \in \bar{O}_{1} \cup \bar{O}_{2}$ if and only if $t=-1$ or $t=1$; finally, $\omega(P)=\sup _{x \in P} \operatorname{dist}(x, \partial P)$ is called the width of $P$.

Theorem 3.1. Let $\left\{\Omega_{i}\right\}_{i=1, \ldots, n}$ be $n$ bounded domains in $\mathbb{R}^{2}$, each homeomorphic to a ball, with smooth boundaries and disjoint closures, $\bar{\Omega}_{i} \cap \bar{\Omega}_{j}=\emptyset$ for every $i \neq j$, and let $f$ be as above. Let $\phi_{i}$ be smooth boundary data,

$$
\phi_{i}: \partial \Omega_{i} \rightarrow \mathbb{R}^{d} \text { for } i=1,2, \ldots, n,
$$

such that for each $i$ the functional $\mathcal{F}\left(\cdot, \Omega_{i}\right)$ has at least two isolated local minimizers in the class

$$
\left\{u \in W^{1,2}\left(\Omega_{i} ; \mathbb{R}^{d}\right),\left.u\right|_{\partial \Omega_{i}}=\phi_{i}\right\}
$$

Then there exists a smooth bounded domain $A \subset \mathbb{R}^{2}$, homeomorphic to a ball and containing the set $\Omega=\cup_{i=1}^{n} \Omega_{i}$, and a boundary value $\phi: \partial A \rightarrow \mathbb{R}^{d}$ such that the functional $\mathcal{F}(\cdot, A)$ has at least $2^{n}$ local minimizers in the class of functions $u \in W^{1,2}\left(A ; \mathbb{R}^{d}\right)$ with $\left.u\right|_{\partial A}=\phi$.

Remark 3.2. We will apply this theorem to the functionals built up in the previous section, hence in the applications the dimension of the target space will always be $d=2$ or $d=3$.

Proof. Step 1. Construction of the set $A$ and of the boundary value.

For each $i=1, \ldots, n-1$ consider smooth embedded curves $\Gamma_{i}$ connecting $\Omega_{i}$ and $\Omega_{i+1}$ transversally such that they are disjoint from the other $\Omega_{j}$, that is $\Gamma_{i} \cap \Omega_{j}=\emptyset$ for every $j \neq i, i+1$.

To construct the domain $A$, consider a sequence of unions of bridges, say $\left\{P^{k}=\cup_{i=1}^{n-1} P_{i}^{k}\right\}_{k \in \mathbb{N}}$, where $P_{i}^{k}$ is a bridge along $\Gamma_{i}$, whose sum of widths is infinitesimal, $\omega_{k}=\sum_{i=1}^{n-1} \omega\left(P_{i}^{k}\right) \rightarrow 0$ as $k$ goes to $\infty$.

Let $A^{k}=\Omega \cup P^{k}$ - recall that $\Omega=\cup_{i=1}^{n} \Omega_{i}$. It is not difficult to see that we can choose bridges $P_{i}^{k}$ in such a way that the open sets $A^{k}$ have smooth boundaries; moreover it is easily recognized that $A^{k}$ are homeomorphic to a ball (see Fig. 8 for the case $n=3$ ).

For what concerns the boundary value, let $w: \mathbb{R}^{2} \backslash \Omega \rightarrow \mathbb{R}^{d}$ be a smooth compactly supported function such that $w \equiv \phi_{i}$ on $\partial \Omega_{i}$ for every $i$, (clearly there is no problem for the existence of such a function), and set $\phi \equiv w$ in $\partial A^{k}$. 


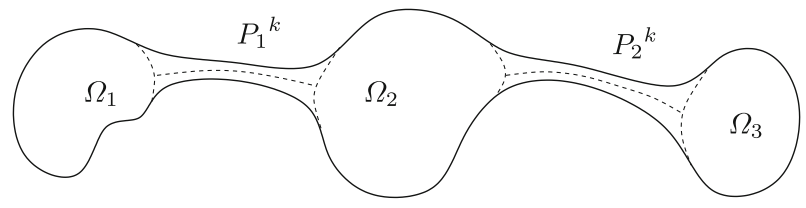

Fig. 8. Example of $A^{k}$ in the case $n=3$

STEP 2. We want to prove that for some $k$ the set $A^{k}$ and the boundary values $\phi \equiv w$ on $\partial A^{k}$ satisfy the thesis of the theorem.

Let $v_{i}^{1}$ and $v_{i}^{2}$ be two isolated local minimizers for $\mathcal{F}\left(\cdot, \Omega_{i}\right)$ (whose existence is guaranteed by hypotheses), $i=1, \ldots, n$, and let $\varepsilon>0$ be such that

$$
\varepsilon<\frac{1}{2} \min \left\{\left\|v_{i}^{1}-v_{i}^{2}\right\|_{W^{1,2}\left(\Omega_{i} ; \mathbb{R}^{d}\right)}, i=1 \ldots, n\right\},
$$

and, for $j=1,2$ and $i=1, \ldots, n$,

$$
\mathcal{F}\left(v_{i}^{j}, \Omega_{i}\right)<\mathcal{F}\left(u, \Omega_{i}\right) \quad \forall u \text { such that }\left\|u-v_{i}^{j}\right\|_{W^{1,2}\left(\Omega_{i} ; \mathbb{R}^{d}\right)} \leqq \varepsilon .
$$

For each $n$-tuple $\alpha=\left(\alpha_{1}, \ldots, \alpha_{n}\right) \in\{1,2\}^{n}$, we consider the problem

$$
\left(\mathcal{P}_{k}^{\alpha}\right) \quad \min \left\{\mathcal{F}\left(u, A^{k}\right): \begin{array}{c}
u \in W^{1,2}\left(A^{k} ; \mathbb{R}^{d}\right),\left.u\right|_{\partial A^{k}} \equiv w, \\
\left\|u-v_{i}^{\alpha_{i}}\right\|_{W^{1,2}\left(\Omega_{i} ; \mathbb{R}^{d}\right)} \leqq \varepsilon, i=1, \ldots, n
\end{array}\right\} .
$$

Note that the sets of functions satisfying the constraints of these problems are not empty: for example they contain the functions $u^{\alpha}$ defined in (3.4).

By the semicontinuity of the functional (see for example [1]) and its coercivity, given by (3.1),

$$
c \int_{A^{k}}|\nabla u(x)|^{2} \mathrm{~d} x \leqq \int_{A^{k}} f(\nabla u(x)) \mathrm{d} x,
$$

the above problems have at least a solution $u_{k}^{\alpha}$. Moreover, fixing $k$, these solutions are all distinct (due to the constraints $\left\|u_{k}^{\alpha}-v_{i}^{\alpha_{i}}\right\|_{W^{1,2}\left(\Omega_{i} ; \mathbb{R}^{d}\right)} \leqq \varepsilon$ for every $i=1, \ldots, n)$.

If we proved that, for some $k$, every $u_{k}^{\alpha}$ satisfies

$$
\left\|u_{k}^{\alpha}-v_{i}^{\alpha_{i}}\right\|_{W^{1,2}\left(\Omega_{i} ; \mathbb{R}^{d}\right)}<\varepsilon, \quad \text { for every } i=1, \ldots, n,
$$

then $\left\{u_{k}^{\alpha}\right\}_{\alpha \in\{1,2\}^{n}}$ would be local minimizers for $\mathcal{F}\left(\cdot, \mathcal{A}^{k}\right)$ in $W^{1,2}\left(A^{k} ; \mathbb{R}^{d}\right)$, thus showing that for such $k$ the domain $A^{k}$ and the boundary value $\phi \equiv w$ on $\partial A^{k}$ provide an example with $2^{n}$ different local minimizers.

STEP 3. Proof of (3.3).

For each $\alpha$, let $u^{\alpha}$ and $u_{k}^{\alpha}$ denote the following functions in $W^{1,2}\left(\mathbb{R}^{2} ; \mathbb{R}^{d}\right)$,

$$
u^{\alpha}=\left\{\begin{array}{ll}
v_{i}^{\alpha_{i}} & \text { in } \Omega_{i}, \quad i=1, \ldots, n \\
w & \text { in } \mathbb{R}^{2} \backslash \Omega ;
\end{array} \quad \text { and } u_{k}^{\alpha}= \begin{cases}u_{k}^{\alpha_{i}} & \text { in } A^{k} \\
w & \text { in } \mathbb{R}^{2} \backslash A^{k}\end{cases}\right.
$$


If we showed that a subsequence of $u_{k}^{\alpha}$ converges to $u^{\alpha}$ in $W^{1,2}\left(\mathbb{R}^{2} ; \mathbb{R}^{d}\right)$ for every $\alpha$, then (3.3) would be proved and the proof concluded.

Fix $\alpha$. First we notice that $\left\{u_{k}^{\alpha}\right\}$ converges weakly to $u^{\alpha}$. Indeed, by the boundedness in $W^{1,2}\left(\mathbb{R}^{2} ; \mathbb{R}^{d}\right.$ ) (because $u_{k}^{\alpha}$ have equibounded energies, as can be seen by the comparison with $u^{\alpha}$ ) the sequence $\left\{u_{k}^{\alpha}\right\}$ converges weakly to some $v \in$ $W^{1,2}\left(\mathbb{R}^{2} ; \mathbb{R}^{d}\right)$. It is readily recognized that $v \equiv w$ in $\mathbb{R}^{2} \backslash \Omega$. By the weak lower semicontinuity of $\mathcal{F}$ we get

$$
\begin{aligned}
\mathcal{F}\left(v, \mathbb{R}^{2}\right) & =\mathcal{F}(v, \Omega)+\mathcal{F}\left(v, \mathbb{R}^{2} \backslash \Omega\right) \leqq \liminf _{k \rightarrow+\infty} \mathcal{F}\left(u_{k}^{\alpha}, \mathbb{R}^{2}\right) \\
& \leqq \liminf _{k \rightarrow+\infty} \mathcal{F}\left(u_{k}^{\alpha}, A^{k}\right)+\mathcal{F}\left(w, \mathbb{R}^{2} \backslash \Omega\right) \\
& \leqq \liminf _{k \rightarrow+\infty} \mathcal{F}\left(u^{\alpha}, A^{k}\right)+\mathcal{F}\left(w, \mathbb{R}^{2} \backslash \Omega\right) \\
& \leqq \liminf _{k \rightarrow+\infty}\left[\mathcal{F}\left(u^{\alpha}, \Omega\right)+\mathcal{F}\left(w, P^{k}\right)\right]+\mathcal{F}\left(w, \mathbb{R}^{2} \backslash \Omega\right) \\
& =\mathcal{F}\left(u^{\alpha}, \Omega\right)+\mathcal{F}\left(w, \mathbb{R}^{2} \backslash \Omega\right)=\mathcal{F}\left(u^{\alpha}, \mathbb{R}^{2}\right),
\end{aligned}
$$

In the above calculation we used the minimality of $u_{k}^{\alpha}$, passing from the second line to the third, and $\mathcal{L}^{2}\left(P^{k}\right) \rightarrow 0$ (because the widths $\omega_{k} \rightarrow 0$ ) in order to deduce the last equalities.

Given that $u^{\alpha}$ is an isolated local minimizer in $\Omega_{i}$ for every $i$, see (3.2), we conclude that $v=u^{\alpha}$.

Next we show that the convergence is strong. A preliminary step is to prove that there exists convergence of the energies:

$$
\lim _{k \rightarrow+\infty} \int_{\mathbb{R}^{2}} f\left(\nabla u_{k}^{\alpha}(x)\right) \mathrm{d} x=\int_{\mathbb{R}^{2}} f\left(\nabla u^{\alpha}(x)\right) \mathrm{d} x .
$$

Indeed, using the minimality of $u_{k}^{\alpha}$ in $A_{k}$, we get

$$
\begin{aligned}
\int_{\mathbb{R}^{2}} f\left(\nabla u_{k}^{\alpha}(x)\right) \mathrm{d} x & =\int_{A^{k}} f\left(\nabla u_{k}^{\alpha}(x)\right) \mathrm{d} x+\int_{\mathbb{R}^{2} \backslash A^{k}} f(\nabla w(x)) \mathrm{d} x \\
& \leqq \int_{A^{k}} f\left(\nabla u^{\alpha}(x)\right) \mathrm{d} x+\int_{\mathbb{R}^{2} \backslash A^{k}} f(\nabla w(x)) \mathrm{d} x \\
& =\int_{\mathbb{R}^{2}} f\left(\nabla u^{\alpha}(x)\right) \mathrm{d} x .
\end{aligned}
$$

Hence, taking into account the lower semicontinuity of $\mathcal{F}$,

$$
\begin{aligned}
\int_{\mathbb{R}^{2}} f\left(\nabla u^{\alpha}(x)\right) \mathrm{d} x & \leqq \liminf _{k \rightarrow+\infty} \int_{\mathbb{R}^{2}} f\left(\nabla u_{k}^{\alpha}(x)\right) \mathrm{d} x \\
& \leqq \limsup _{k \rightarrow+\infty} \int_{\mathbb{R}^{2}} f\left(\nabla u_{k}^{\alpha}(x)\right) \mathrm{d} x \\
& \leqq \int_{\mathbb{R}^{2}} f\left(\nabla u^{\alpha}(x)\right) \mathrm{d} x .
\end{aligned}
$$

Using this convergence of the energies and the strict polyconvexity of the integrand $f(x)=c|x|^{2}+g(x$, det $x)$, we can prove the strong convergence. Indeed, because 
of the polyconvexity of $g(x, \operatorname{det} x)=f(x)-c|x|^{2}$ and hence the semicontinuity of the related functional, we have

$$
\begin{aligned}
& \int_{\mathbb{R}^{2}}\left[f\left(\nabla u^{\alpha}(x)\right)-c\left|\nabla u^{\alpha}(x)\right|^{2}\right] \mathrm{d} x \\
& =\int_{\mathbb{R}^{2}} g\left(\nabla u^{\alpha}(x), \operatorname{det} \nabla u^{\alpha}(x)\right) \mathrm{d} x \\
& \quad \leqq \liminf _{k \rightarrow+\infty} \int_{\mathbb{R}^{2}} g\left(\nabla u_{k}^{\alpha}(x), \operatorname{det} \nabla u_{k}^{\alpha}(x)\right) \mathrm{d} x \\
& =\liminf _{k \rightarrow+\infty} \int_{\mathbb{R}^{2}}\left[f\left(\nabla u_{k}^{\alpha}(x)\right)-c\left|\nabla u_{k}^{\alpha}(x)\right|^{2}\right] \mathrm{d} x .
\end{aligned}
$$

Thus, by (3.5),

$$
\limsup _{k \rightarrow+\infty} \int_{\mathbb{R}^{2}}\left|\nabla u_{k}^{\alpha}(x)\right|^{2} \mathrm{~d} x \leqq \int_{\mathbb{R}^{2}}\left|\nabla u^{\alpha}(x)\right|^{2} \mathrm{~d} x .
$$

Now it is easily recognized that (3.6) and the weak convergence $u_{k}^{\alpha} \rightarrow u^{\alpha}$ in $W^{1,2}\left(\mathbb{R}^{2} ; \mathbb{R}^{d}\right)$ give the strong convergence in $W^{1,2}\left(\mathbb{R}^{2} ; \mathbb{R}^{d}\right)$, thus concluding the proof.

Remark 3.3. We point out that the above proof can be obviously generalized in the case $\mathcal{F}\left(\cdot, \Omega_{i}\right)$ has $k_{i}$ isolated local minimizers (instead of 2); the theorem then provides a domain with $\prod_{i=1}^{n} k_{i}$ local minimizers.

Remark 3.4. We recall that the argument about strong convergence of weak convergent sequences in the presence of the convergence of quasiconvex energy was given by Evans and Gariepy in [6] and [7]. We thank Prof. Nicola Fusco for pointing out these references.

We can, hence, consider the case of the functional $\mathcal{F}$ of Theorem 1.1 and prove Theorem 1.2.

Proof of Theorem 1.2. We showed in Theorem 1 that there exists a boundary value problem for $\mathcal{F}$ with at least two smooth (analytic) absolute minimizers: if they were isolated we could apply Theorem 3.1 to

$$
\Omega_{i}=(3 i, 0)+D \quad i=1, \ldots, n,
$$

the translated by the vector $(3 i, 0)$ of the unit disc $D$, and

$$
\phi_{i}(x)=u_{0}(x-(3 i, 0))
$$

as boundary data (see Fig. 9 in the case $n=3$ ). In this way we conclude the existence of a pure displacement boundary value problem for a strict polyconvex functional defined on a set homeomorphic to a ball with at least $2^{n}$ local minimizers (Fig. 10).

On the other hand, if $\mathcal{F}$ does not have two isolated absolute minimizers, it is readily recognized that it must have uncountably many minimizers (because the set of absolute minimizers would be a perfect set, that is, would coincide with the set of its limit points), having in any case an example with arbitrarily many minimizers. Thus proves Theorem 1.2. 


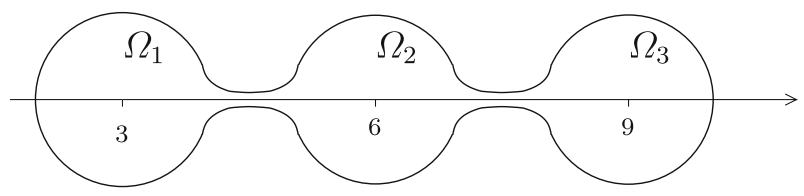

Fig. 9. Example of domain with $2^{3}$ local minimizers

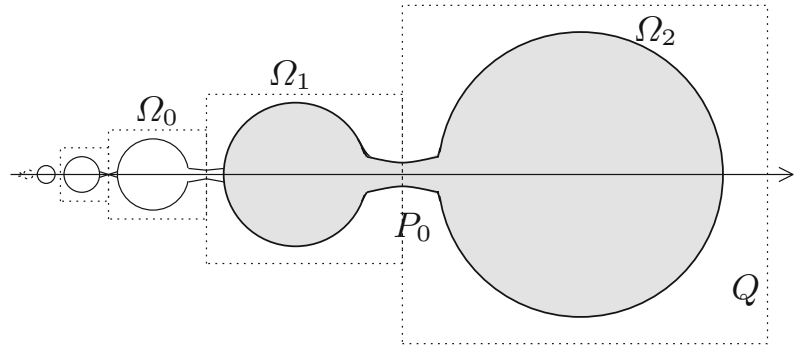

Fig. 10. Construction of the set $A_{i}$ : the dashed one is $A_{0}$

Remark 3.5. In the same way, if we consider functions with target $\mathbb{R}^{3}$, we can also manage the boundary value to be one to one (using Theorem $1.1^{\prime}$ ).

With the techniques in Theorem 3.1 we may also exhibits an example of a strictly polyconvex boundary value problem with uncountably many stable equilibrium solutions.

Proof of Theorem 1.3. The idea is to iterate the construction in Theorem 1.2. Consider the functional $\mathcal{F}$ given in Theorem 1.1.

STEP 1 . We construct the set $A$ and the boundary value.

Let us define the vectors $a_{i}=\left(2^{-i}, 0\right)$. Consider the domains

$$
\Omega_{i}=a_{i}+2^{-i-2} D, \quad \text { for } i \in \mathbb{N}
$$

which are translated and rescaled unit discs; let $\Omega=\cup_{i \in \mathbb{N}} \Omega_{i}$, and

$$
\phi_{i}(x)=2^{-i-2} u_{0}\left(2^{i+2}\left(x-a_{i}\right)\right), \quad \text { for } x \in \partial \Omega_{i},
$$

with $u_{0}$ the same as in Theorem 1.1. It is clear that $\mathcal{F}\left(\cdot, \Omega_{i}\right)$ still has two smooth absolute minimizers $v_{i}^{1}$ and $v_{i}^{2}$ with boundary value $\phi_{i}$.

Now consider a function $w \in C_{c}\left(\mathbb{R}^{2} \backslash \Omega ; \mathbb{R}^{2}\right) \cap W^{1,2}\left(\mathbb{R}^{2} \backslash \Omega ; \mathbb{R}^{2}\right)$ such that $w \equiv \phi_{i}$ on $\partial \Omega_{i}$.

It is not difficult to show the existence of such a function. Consider for example a smooth extension of $\phi_{0}$ in $\mathbb{R}^{2} \backslash \Omega_{0}$ with support in $Q$, where $Q$ is the cube centered at $(1,0)$ with side length $\frac{3}{8}$. We define $w(x)=\sum_{i \in \mathbb{N}} 2^{-i-2} \phi_{0}\left(2^{i+2}\left(x-a_{i}\right)\right)$ and with an easy calculation see that $w$ satisfies the hypotheses - see Fig. 10.

Now we can start our recursive procedure: as usual we can suppose that the minimizers of $\mathcal{F}\left(\cdot, \Omega_{i}\right)$ are isolated (otherwise there is nothing to prove). 
By Theorem 3.1 there exists a bridge $P_{0}$ connecting $\Omega_{0}$ and $\Omega_{1}$ such that, for the smooth open set $A_{0}=\Omega_{0} \cup P_{0} \cup \Omega_{1}$ and for the boundary value $\phi \equiv w$ on $\partial A_{0}$, the functional $\mathcal{F}$ has at least four local minimizers,

$$
u^{\left(\alpha_{0}, \alpha_{1}\right)} \quad \text { with } \quad \alpha_{i} \in\{1,2\} .
$$

Moreover these minimizers are close to the original minimizers in $W^{1,2}$-norm,

$$
\left\|u^{\left(\alpha_{0}, \alpha_{1}\right)}-v_{i}^{\alpha_{i}}\right\|_{W^{1,2}\left(\Omega_{i} ; \mathbb{R}^{2}\right)} \leqq \varepsilon_{1} \quad \text { for } i=0,1,
$$

for some $\varepsilon_{1}>0$ to be chosen later.

Again, we may suppose that these minimizers are isolated and use Theorem 3.1 to conclude that there exists a bridge $P_{1}$ connecting $A_{0}$ and $\Omega_{2}$ such that in $A_{1}=A_{0} \cup P_{1} \cup \Omega_{2}$ we have at least eight local minimizers for $\mathcal{F}$ with boundary value $w$, and such minimizers are close in norm to the previous one, so

$$
\left\|u^{\left(\alpha_{0}, \alpha_{1}, \alpha_{2}\right)}-u^{\left(\alpha_{0}, \alpha_{1}\right)}\right\|_{W^{1,2}\left(A_{0} ; \mathbb{R}^{2}\right)} \leqq \varepsilon_{2},
$$

and

$$
\left\|u^{\left(\alpha_{0}, \alpha_{1}, \alpha_{2}\right)}-v_{2}^{\alpha_{2}}\right\|_{W^{1,2}\left(\Omega_{2} ; \mathbb{R}^{2}\right)} \leqq \varepsilon_{2}
$$

where every $\alpha_{i}$ ranges over the set $\{1,2\}$, and for some $\varepsilon_{2}>0$.

Repeating this argument, we obtain a sequence of smooth open sets $A_{k}$ and a sequence of functions $\left\{u^{\alpha^{k}}\right\}_{k \in \mathbb{N}}^{\alpha^{k} \in\{1,2\}^{k}}$ which are local minimizers in $A_{k}$ with boundary value $w$, and such that

$$
\left\|u^{\left(\alpha_{0}, \ldots, \alpha_{k+1}\right)}-u^{\left(\alpha_{1}, \ldots, \alpha_{k}\right)}\right\|_{W^{1,2}\left(A_{k-1} ; \mathbb{R}^{2}\right)} \leqq \varepsilon_{k}
$$

and

$$
\left\|u^{\left(\alpha_{0}, \ldots, \alpha_{k+1}\right)}-v_{k}^{\alpha_{k+1}}\right\|_{W^{1,2}\left(\Omega_{k} ; \mathbb{R}^{2}\right)} \leqq \varepsilon_{k},
$$

where $\varepsilon_{k}>0$ are to be fixed.

In order to do that, let $\delta_{m}$ be such that

$$
\left\|v_{m}^{1}-v_{m}^{2}\right\|_{W^{1,2}\left(\Omega_{m} ; \mathbb{R}^{2}\right)}<\frac{\delta_{m}}{2} .
$$

We choose $\varepsilon_{k}$ in such a way that

$$
\sum_{k=m}^{\infty} \varepsilon_{k} \leqq \delta_{m} \quad \forall m \in \mathbb{N} .
$$

Consider the functions $u^{\alpha^{k}}$ defined in all of $\mathbb{R}^{2}$, being extended out of the respective $A_{k}$ by $w$.

For every infinite sequence $\alpha=\left(\alpha_{1}, \alpha_{2}, \ldots\right) \in\{1,2\}^{\aleph_{0}}$, consider the sequence $\left\{u^{\alpha_{k}}\right\}$, where $\alpha_{k}=\left(\alpha_{1}, \ldots, \alpha_{k}\right)$ is the $k$-tuple of the first $k$ entries of $\alpha$. This is 
a convergent sequence - because of (3.7) and (3.10) - and we denote its limit by $u^{\alpha}$. For distinct $\alpha$ and $\beta$ the limits $u^{\alpha}$ and $u^{\beta}$ are different, thanks to the conditions (3.8), (3.10) and (3.9).

So, we have obtained a set of distinct functions $\left\{u^{\alpha}\right\}_{\alpha \in\{1,2\}^{\aleph_{0}}}$ such that $u^{\alpha} \equiv w$ on $\partial A$, where

$$
A=\cup_{k=0}^{\infty} A_{k}=\lim _{k \rightarrow+\infty} A_{k}
$$

Note that $A_{k} \subset A_{k+1}$ and that $A$ is homeomorphic to a ball, with $\partial A$ lipschitz regular except that at the origin $(0,0)$.

STEP 2. Finally, we prove that the above $A$ and the boundary value $w$ on $\partial A$ have the desired property.

Since they are limits of local minimizers, hence in particular stable equilibrium solutions, it follows that the $u^{\alpha}$ are stable equilibrium points for $\mathcal{F}$. Indeed, for every $\varphi \in C_{c}^{\infty}(A)$ it must be $\operatorname{supp} \varphi \subset A_{j}$, for some $j$, and for every $u^{\alpha}$ we have

$$
\begin{aligned}
\frac{d}{d \varepsilon} \mathcal{F}\left(u^{\alpha}+\varepsilon \varphi\right)_{\mid \varepsilon=0} & =\int_{A} \nabla f\left(\nabla u^{\alpha}(x)\right) \cdot \nabla \varphi(x) \mathrm{d} x \\
& =\int_{A_{j}} \nabla f\left(\nabla u^{\alpha}(x)\right) \cdot \nabla \varphi(x) \mathrm{d} x \\
& =\lim _{k \rightarrow+\infty} \int_{A_{j}} \nabla f\left(\nabla u^{\alpha^{k}}(x)\right) \cdot \nabla \varphi(x) \mathrm{d} x=0,
\end{aligned}
$$

because $u^{\alpha^{k}}$ converges in norm to $u^{\alpha}$ and are all stationary points in $A_{j}$ for $k \geqq j$.

Analogously, for the stability we have

$$
\begin{aligned}
\frac{\mathrm{d}^{2}}{\mathrm{~d} \varepsilon^{2}} \mathcal{F}\left(u^{\alpha}+\varepsilon \varphi\right)_{\mid \varepsilon=0} & =\int_{A} D^{2} f\left(\nabla u^{\alpha}(x)\right) \nabla \varphi(x) \cdot \nabla \varphi(x) \mathrm{d} x \\
& =\int_{A_{j}} D^{2} f\left(\nabla u^{\alpha}(x)\right) \nabla \varphi(x) \cdot \nabla \varphi(x) \mathrm{d} x \\
& =\lim _{k \rightarrow+\infty} \int_{A_{j}} D^{2} f\left(\nabla u^{\alpha^{k}}(x)\right) \nabla \varphi(x) \cdot \nabla \varphi(x) \mathrm{d} x=0,
\end{aligned}
$$

that is $u^{\alpha}$ are stable for perturbations in $C_{c}^{\infty}(A)$. Moreover, since $A$ has smooth boundary except at 0 , it is not difficult to show that $C_{c}^{\infty}\left(A ; \mathbb{R}^{2}\right)$ is dense in $W_{0}^{1,2}\left(A ; \mathbb{R}^{2}\right)$, so that from the previous calculation follows the stability for perturbations $\phi \in W_{0}^{1,2}\left(A ; \mathbb{R}^{2}\right)$.

So, $\left\{u^{\alpha}\right\}_{\alpha \in\{1,2\}^{\aleph_{0}}}$ being all distinct stationary equilibrium solutions, we have proved the theorem.

Remark 3.6. As usual, the same result is true with one to one boundary value in the three dimensional case (we need only to start the procedure from a functional with injective boundary data, see Theorem $1.1^{\prime}$ ). 


\section{References}

1. Acerbi, E., Fusco, N.: Semicontinuity problems in the calculus of variations. Arch. Ration. Mech. Anal. 86(2), 125-145 (1984)

2. BALl, J.M.: Discontinuous equilibrium solutions and cavitation in nonlinear elasticity. Philos. Trans. R. Soc. Lond. Ser. A, 306(1496), 557-611 (1982)

3. BALL, J.M.: Some open problems in elasticity. In: Geometry, Mechanics, and Dynamics. Springer, New York, 3-59, 2002

4. Dierkes, U., Hildebrandt, S., Küster, A., Wohlrab, O.: Minimal surfaces. I, vol 295 of Grundlehren der Mathematischen Wissenschaften [Fundamental Principles of Mathematical Sciences]. Springer, Berlin, 1992. Boundary value problems

5. Dierkes, U., Hildebrandt, S., Küster, A., Wohlrab, O.: Minimal surfaces. II, vol 296 of Grundlehren der Mathematischen Wissenschaften [Fundamental Principles of Mathematical Sciences]. Springer, Berlin, 1992. Boundary regularity

6. Evans, L.C., GariePY, R.F.: Blowup, compactness and partial regularity in the calculus of variations. Indiana Univ. Math. J. 36(2), 361-371 (1987)

7. Evans, L.C., GARIEPY, R.F.: Some remarks concerning quasiconvexity and strong convergence. Proc. R. Soc. Edinb. Sect. A 106(1-2), 53-61 (1987)

8. Evans, L.C.: Weak convergence methods for nonlinear partial differential equations, vol. 74 of CBMS Regional Conference Series in Mathematics. Published for the Conference Board of the Mathematical Sciences, Washington, DC, 1990

9. Hildebrandt, S.: Boundary behavior of minimal surfaces. Arch. Ration. Mech. Anal. 35, 47-82 (1969)

10. JoHN, F.: Uniqueness of non-linear elastic equilibrium for prescribed boundary displacements and sufficiently small strains. Comm. Pure Appl. Math., 25, 617-634 (1972)

11. Knops, R.J., Stuart, C.A.: Quasiconvexity and uniqueness of equilibrium solutions in nonlinear elasticity. Arch. Ration. Mech. Anal. 86(3), 233-249 (1984)

12. Kristensen, J., TAheri, A.: Partial regularity of strong local minimizers in the multidimensional calculus of variations. Arch. Ration. Mech. Anal. 170(1), 63-89 (2003)

13. LaWson, H.B. JR., OsSERman, R.: Non-existence, non-uniqueness and irregularity of solutions to the minimal surface system. Acta Math. 139(1-2), 1-17 (1977)

14. Lloyd, N.G.: Degree theory. Cambridge University Press, Cambridge, 1978. Cambridge Tracts in Mathematics, No. 73

15. MÜLler, S., ŠverÁK, V.: Convex integration for Lipschitz mappings and counterexamples to regularity. Ann. of Math. (2) 157(3), 715-742 (2003)

16. PiPKIn, A.C.: Convexity conditions for strain-dependent energy functions for membranes. Arch. Ration. Mech. Anal. 121(4), 361-376 (1992)

17. Post, K.D.E., Sivaloganathan, J.: On homotopy conditions and the existence of multiple equilibria in finite elasticity. Proc. R. Soc. Edinburgh Sect. A, 127(3), 595-614 (1997)

18. SzÉKelyhidi, L. JR.: The regularity of critical points of polyconvex functionals. Arch. Ration. Mech. Anal. 172(1), 133-152 (2004)

19. TAHERI, A.: On critical points of functionals with polyconvex integrands. J. Convex Anal. 9(1), 55-72 (2002)

20. TAHERI, A.: Quasiconvexity and uniqueness of stationary points in the multi-dimensional calculus of variations. In: Proceedings of the American Mathematical Society 131(10), 3101-3107 (electronic) (2003)

21. TAHERI, A.: Local minimizers and quasiconvexity-the impact of topology. Arch. Ration. Mech. Anal. 176(3), 363-414 (2005)

22. White, B.: The bridge principle for stable minimal surfaces. Calc. Var. Partial Differ. Equ. 2(4), 405-425 (1994) 
Institut für Mathematik, Universität Zürich, 8057 Zürich, Switzerland. e-mail: emanuele.spadaro@math.unizh.ch

(Received July 16, 2007 / Accepted March 4, 2008)

Published online August 5, 2008 - (C) Springer-Verlag (2008) 